\title{
Formulation of Two-Stage Problem of Structural- Parametric Synthesis of Adaptive Electronic Document Management System
}

\author{
Artem Obukhov ${ }^{1}$, Mikhail Krasnyanskiy ${ }^{2}$, and Denis Dedov ${ }^{3}$ \\ ${ }^{1}$ Department of Automated Decision Support Systems, Tambov State Technical University, Russian Federation \\ ${ }^{2}$ Department of Administration, Tambov State Technical University, Russian Federation \\ ${ }^{3}$ Department of Science, Tambov State Technical University, Russian Federation
}

\begin{abstract}
The paper scrutinizers the options of optimization and adaption to the individual characteristics of users of Electronic Document Management Systems (EDMS). The problem solution requires further development of the necessary methods, models and criteria. Therefore, the article considers the problem of the structural-parametric synthesis of adaptive EDMS. On the basis of previously conducted research in this area, a new architecture of EDMS is developed, within which a mathematical model of adaptive EDMS is proposed. It includes the main components of the information system, as well as a set of estimates for a number of indicators: total discounted costs, productivity, software quality and, above all, adaptability to the requirements of the user. Using this mathematical model, a two-stage task of structural-parametric synthesis of an adaptive EDMS was set, at the first stage of which the system is synthesized according to the criterion of economic efficiency, and at the second stage the process happens according to its adaptation to each user. The scientific novelty of the proposed approach consists in dividing the optimization task into two stages such as the formalization of the criteria for adaptive EDMS and development of a new architecture and mathematical model of adaptive EDMS. The results can be used to solve problems of design, modernization and adaptation of various information systems.
\end{abstract}

Keywords: Adaptability, electronic document management systems, optimization problem statement, structural-parametric synthesis.

\section{Introduction}

Modern information systems are becoming more personalized every year, adapted to a wide range of software platforms and operating systems. Electronic Document Management Systems (EDMS) are also developing in this direction, becoming more and more flexible, adapted and cross-platform $[1,7,11]$.

The problem of user-friendly, adapted interfaces development for users is relevant, since there are contradictions between the developer's opinion about effective interface and the subjective wishes of users [1]. The new ways of interaction with the interface and software and hardware platforms, increasing device performance, leads to the information systems modernization $[1,6,12]$.

We also note that the company's business processes are also in constant development and change, which requires a corresponding modification of the information system, including the interface. The automation of this process would significantly reduce the time and material costs in the process of information system development and upgrade. The comparative analysis of approaches outlined in [1] showed that the only and universal solution for interfaces construction is not presented. Some of the methods are effective only at the design stage, and part of them allows formalizing the requirements for the interface in detail, but does not allow constructing.

Thus, the aim of the study consists in the increase of EDMS adaptability through the development of new methods, models and criteria.

This article considers the formulation of the problem of structural-parametric synthesis of adaptive EDMS, the solution of which is aimed at improving the quality, efficiency and reliability of such information systems, as well as optimizing the system for individual user characteristics.

\section{Materials and Methods}

\subsection{Related Works}

Considering the general approaches to the problem formulation of structural-parametric synthesis, at the first stage it is necessary to choose the appropriate mathematical apparatus which allows you to formalize, set and solve the optimization problem. In the course of analyzing approaches to workflow processes modeling, the main types of models were identified:

1. Set-theoretic.

2. Graph-theoretic. 


\section{Automatic. \\ 4. Functional. \\ 5. Descriptor. \\ 6. Multi-agent, and their strengths and weaknesses are defined $[11,13,14]$.}

The graph-theoretical model was chosen as the basis for the mathematical model of EDMS [10, 11]. The selection of this model is reflected by its ability to strictly formalize the structure of EDMS and the relationship between the system components, the processes of data transmission and processing. Also, this model made it possible to apply and adapt the results, obtained by the authors earlier in this area. When implementing the model, it is necessary to take into account the internal structure of the document, the operations carried out on them, the software, hardware and structural parameters of the information system, as well as factors affecting the adaptation of EDMS to the user's needs [15].

When implementing this mathematical model, the previously obtained results on problems solving of the structural-parametric synthesis of EDMS of a scientific educational institution will be used [11]. In developed mathematical model of an adaptive EDMS it is necessary to integrate provisions regarding interface evaluation [23], as well as to consider the possibility of increasing the flexibility of the information system through the use of machine learning methods. The latter repeatedly proved their effectiveness in the tasks of information processing; however, they have not yet been used directly in solving the problems of the synthesis of EDMS.

For the problem formulation of adaptive EDMS synthesis it is required to analyze the approaches to the practical implementation of the possibility of adapting the interface of information systems, as well as theoretical methods for estimation of this parameter [16].

There are many different approaches to adapting the interface, but they are often limited to a narrow area of the problem, solved by developers. These approaches can't be scaled or formalized. For example, in [21] algorithmic support of interface adaptation is presented, but its low level of detail does not allow applying it in the subject area of EDMS.

An important part of the problem solution for the adaptation is the study of the properties of the user's entity in the information system, which is reflected in $[4,22]$. It discusses several approaches for information collection about the user, evaluation his equipment and used software.

It is possible to evaluate the adaptability of the interface using a mathematical model of the strategy for building the interface described in [23]. The authors also presented an adaptive man-machine interface algorithm, based on a collection of user's information and subsequent system adaptation, and interface evaluation criteria: the time from the moment of accessing the system; functionality; flexibility; interface security against errors; availability; comfort. The set of criteria affects two indicators that are important for the interface: the ability of the user to adapt to the options of the system and the ability to adapt the system to the individual characteristics of the user. Further, the authors propose a method for calculating these indicators, study the patterns of influence of criteria on adaptation indicators, and then formulate a methodology for selecting an adaptive interface structure using a variety of evaluation criteria. We note the high scientific and practical value of this work and the ability to integrate the provisions outlined in it within the task set for the synthesis of an adaptive EDMS.

A promising direction in adapting the interface to the user's individual characteristics is the use of machine learning methods, for example, neural networks $[5,9,20]$.

For example, in [5] the authors successfully solved the problem of choosing interface components applying a recommendation system based on machine learning methods. An application was used to monitor the state of the environment as a subject area. The authors implemented a system of component recommendations for new users based on the preferences of already registered users by accomplishing the training of machine learning algorithms based on user data (age, position, and equipment), time of year and part of the day when he interacted with the system. Since the approach, presented in the article, is of high scientific and practical importance and was successfully tested, its individual aspects will be used in solving the task of adapting EDMS and developing the necessary algorithmic support.

Summarizing the conducted analysis, we note that there is no formalized representation of the interface adaptation algorithms in most of the works, but there is only their verbal description. In [23], the necessary mathematical software for assessing the adaptability of the interface and the method of its evaluation are presented. However, the provisions outlined in it must be adapted to the subject area of EDMS for use in problem solving of structural-parametric synthesis of adaptive EDMS. When solving the problem of structural-parametric synthesis, it is necessary to consider the possibility of using machine learning methods [2, 8, 18], since this tool is becoming increasingly common in solving problems of automation, analysis and synthesis, shows high efficiency and speed of work. The disadvantages of this approach include the need for a large amount of input data for studying the machine learning algorithms.

The considered models and approaches to EDMS development are aimed at economic costs optimization 
or productivity raise. The assessment of EDMS adaptability degree is not taken into account in them, since the task of simultaneous optimization according to several criteria and system adaptation to users is very difficult. Within the proposed approach, it is planned to single out the adaptation stage as a separate sub-task, which will allow us to achieve the set goal of EDMS personalization increase in comparison with existing models and methods.

\subsection{Requirements for EDMS Architecture}

Based on the conducted analysis of the approaches to the design of adaptive EDMS, it was concluded that, within the framework of the previously developed mathematical model of EDMS of a scientific and educational institution [11], it is impossible to effectively formulate and solve the problem of structural-parametric synthesis. Therefore, it is proposed to revise the existing model in the framework of the new concept of EDMS adaptive architecture, in which the following requirements should be taken into account:

- Isolation and independence of the key subsystems of EDMS, which allows the decomposition of the design task, parallel automated development of system modules, and upgrading individual components without affecting the work of other subsystems.

- Isolation of the components of mathematical software, for example, the separation of mathematical model of processes of information flows movement of electronic and paper workflow from the functional elements of EDMS responsible for solving specific tasks and the implementation of the requirements of the technical task, as well as the separation of information processing and management.

- Separation of models of information processes and functional elements from control systems and information presentation systems, which ensures their efficiency when changing the structure of information flows of an organization and adding new functions, changing characteristics of a user's terminal.

- Independence of control systems and information presentation from data models allows the crossplatform design of EDMS for both stationary and mobile platforms, various operating systems and architectures.

- Consideration of the influence of the user, as a participant of the workflow, on the processes of information flows movement, control and visualization elements of EDMS, which allows formalizing the processes of document routing and adaptation of control and visualization elements to the individual characteristics of the user.
- Option to adapt EDMS structural blocks to the features of the subject area, user terminal parameters without the need to make significant changes to EDMS architecture, disturbance of the performance of the presentation, control and full software processing systems.

The existing mathematical, structural, informational and other EDMS models do not consider the highlighted requirements.

Thus, when solving the problem of structuralparametric synthesis of an adaptive EDMS, it is necessary to implement a new architecture of EDMS, taking into account the listed requirements, the software, on the basis of which the formulation of the task of EDMS will be performed, with the possibility of adapting its interface to the individual characteristics of users.

\section{Results}

We consider the implementation of the proposed architecture, the mathematical model of an adaptive EDMS and the formulation of the problem of its synthesis. The proposed approach has the following distinctive features such as dividing the optimization task into two stages, new EDMS architecture development and formalization of calculation formulas for EDMS adaptation criteria.

\subsection{Adaptive EDMS Architecture}

We mark the proposed EDMS architecture as "UserModel-View-Control" or (UMVC), since 4 components, listed above, are defining in the design of an adaptive EDMS, if we analyze the conditions presented earlier. When implementing this concept, the approaches and techniques, proposed for the implementation of software based on Model-ViewController (MVC) concept [17] were used; however, this paradigm applies not only to software, but to the entire structure of EDMS, including not only software modules, but also their algorithmic and mathematical software, the direct influence of the user and the need to take into account its influence on the structure of EDMS. These fundamental differences of the proposed UMVC concept from MVC and the discrepancy in the areas of their application suggest the originality of the proposed UMVC concept.

The general concept of UMVC architecture in the form of a structural model is presented in Figure 1. The user $U$ is directly involved in the workflow process with the ability to create, process, manage information flows, can act either as an initiator of an operation on documents, or as an observer, performer, manager, etc., Also, each user corresponds to the current software $S W$ and hardware $H W$ of his terminal, which has a significant impact on the features of the operation of EDMS. 


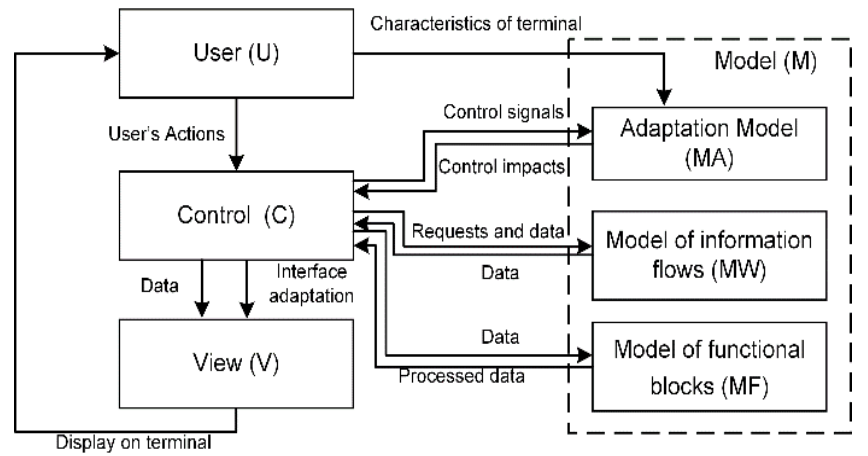

Figure 1. Structural model of adaptive EDMS based on UMVC architecture.

The model will be understood as the mathematical, datalogical or algorithmic support of the components of EDMS, and each model is isolated and cannot disturb the internal structure and contents of the other. This rule ensures the integrity of the data and the possibility of independently design of each model and replacing them with one another. Three models, necessary for its implementation, can be immediately identified from the analysis of the tasks, set for the developed architecture: Information flow model $M W$ (contains data on the structure, processes of movement and interaction of documents, their life cycles), Functional block model $M F$ (contains mathematical relationships that implement certain processing and data conversion into EDMS, aimed at solving specific tasks within the framework of program modules), adaptation model MA (includes the necessary mathematical, algorithmic support and software, which is responsible for the analysis of user equipment, requirements for the composition and design of the interface, as well as the methodology for EDMS evaluating according to various criteria).

The Control $C$ is intended to control and transfer information between Models and User with help of special control blocks that convert and process data according to specified mathematical relationships in such way that the functional blocks can interact with them regardless of the characteristics of the subject area and, therefore, the data structure. In addition, Control is responsible for the interface adaption to the individual features of User.

The View $V$ is a textual, graphical or other display of information for the user, formed by request of control blocks from the data of Information Flow Model using the Function Block Model. The isolation of representation from the remaining blocks is a prerequisite, since it provides the cross-platform and possibility of free modification of interfaces for individual features of client devices and user requirements.

\subsection{Mathematical Model of Adaptive EDMS}

Next, we consider a mathematical model of adaptive EDMS, implemented on the basis of UMVC architecture using the set theory apparatus and allowing solving the problem of structural-parametric synthesis of adaptive EDMS.

In a formalized form the mathematical model has the form:

$$
U M V C \rightarrow(U, V, C, M W, M F, M A)
$$

We consider each of the components in detail.

Every user $u_{q} \in U$ corresponds to a set of software $S W_{q j}$ and the hardware $h w_{q j}$ characteristics of his terminal:

$$
u_{q} \rightarrow\left\{s w_{q j}, h w_{q j}\right\}
$$

The presentation includes a set of elements of visualization $v_{k} \in V$, Control is a set of control blocks $c_{m} \in C$. Their combination forms the structure and parameters of EDMS software modules:

$$
\begin{aligned}
& V \times C \rightarrow C M, \\
& C M=\left\{c m_{i}=\left(S M_{i}, P M_{i}\right)\right\},
\end{aligned}
$$

Where $S M_{i}, P M_{i}$-module structure and parameters $\mathrm{cm}_{i}$.

Data transfer channels between the components are defined by the set $\mathrm{CH}$ :

$$
C H=\left\{c h_{i, j} \mid c m_{i}, c m_{j} \in C M\right\}
$$

The model of Information Flows is based on the application of the model of multilevel graphs $[10,19]$ for the formalization of the processes of movement and interaction of documents. It consists of the following key components:

$$
M W=\{(D, E, O, T, G)\},
$$

Where $D=\left\{d_{a} \mid a=\overline{1 . . n D}\right\}$ - set of objects of document circulation - documents, $n D$-total number of objects; $E$ - set of impacts on objects, both external and internal; $O=\left\{o_{l} \mid l=\overline{1 . . n L}\right\}$ _ set of operations performed on objects, $n L_{-}$their total number; $T$ - set of discrete points in time; $G=\left\{G_{a}\left(d_{a}, O\right) \mid a=\overline{1 . . n G}\right\}$ _ set of structures of information flows, each of which reflects the life cycle of an object $d_{a}$ through the implementation of a set of operations $O$.

The model of functional blocks includes the parameters of input $M D_{I N}$ and output $M D_{\text {OUT }}$ data for functional modules, as well as the software $M P$, required for the operation of the modules, respectively:

$$
M F=\left\{\left(M D_{\text {IN }}, M D_{\text {oUT }}, M P\right)\right\}
$$

Adaptation model includes a set of criteria for EDMS evaluation used at the stage of its design and modernization to obtain the optimal structure and parameters of an information system:

$$
M A=\left(R_{V}, R_{E}, R_{Q}, R_{A}\right),
$$


Where $R_{V}$-evaluation of total discounted costs, reflecting the economic efficiency of the EDMS.

- $R_{E}$ - performance evaluation of EDMS.

- $R_{Q}$ - evaluation of the work quality of EDMS.

- $R_{A}$ - evaluation of adaptability degree of EDMS.

The evaluation of the economic efficiency of EDMS is carried out on the basis of total discounted costs $R_{V}$, therefore, the greatest efficiency will be achieved when choosing EDMS with the lowest total cost. The costs $R_{V}$ are determined by the following equation [11]:

$$
R_{V}=\left(V_{h w}+V_{d}\right)+\sum_{i=1}^{n Y} \frac{v e_{i}}{(1+D R)^{i}}
$$

Where $V_{h w}$-equipment costs (purchase of servers and auxiliary equipment); $V_{d}$-cost of system development (license fees for software, payment of developers); $n Y$ estimated life of EDMS, years; $v e_{i}$-operating costs (electricity, rent, depreciation) in the $i$ year; $D R$ discounted rate, determined by the equation:

$$
D R=\frac{p r+n d+i n}{100}
$$

Where $p r$ - bank interest on long-term deposits; $n d$ calculated increase of the value of the discount rate, in percent; in - expected annual rate of inflation, in percent.

Performance evaluation of EDMS is determined by the number of documents processed over a certain period of time [11]:

$$
R_{E}=\frac{\sum_{i=1}^{K} w_{i}}{\sum_{i=1}^{K} \sum_{j}^{J_{i}} t_{i j} w_{i}},
$$

Where $w_{i}$ - number of documents of $i$ category; $J_{i^{-}}$ number of operations on the document of $i$ category; $t_{i j}$ - time to perform $j$ processing operation of $i$ category document $K$ - total number of categories.

The quality of the work of EDMS is determined by expert assessment by a number of indicators, which can be represented in a formalized form as follows [11]:

$$
R_{Q}=\left\{\left(Q_{i j k}, q_{i j k}\right)\right\}, 0 \leq q_{i j k} \leq 1,
$$

Where $Q_{i j k}-$ set of evaluation elements, the index $i$ corresponds to the factor number, the index $j$ corresponds to the metric number, the index $k$ denotes the ordinal number of the evaluation element, and $q_{i j k}$ shows the value of this evaluation element.

Using a set of evaluation coefficients $\varphi$ :

$$
\sum_{k=1}^{K} \varphi_{j k}=\text { Const }=1
$$

$$
\begin{aligned}
& \sum_{j=1}^{J} \varphi_{i j}=\text { Const }=1 \\
& \sum_{i=1}^{I} \varphi_{i}=\text { Const }=1
\end{aligned}
$$

We receive the final formula to determine the quality assessment:

$$
\begin{gathered}
R_{Q}=\sum_{i=1}^{I}\left(\varphi_{i} \cdot Q_{i}\right), \\
Q^{i}=\sum_{j=1}^{J}\left(\varphi_{i j} \cdot Q_{i j}\right) \\
Q_{i j}=\sum_{k=1}^{K}\left(\varphi_{j k} \cdot q_{i j k}\right)
\end{gathered}
$$

The degree of adaptability of EDMS represents a linear convolution of a set of criteria:

$$
R_{A}=\sum_{i=1}^{6} \lambda_{i} r_{i}(S W, H W)
$$

Where $\lambda_{i}$ - weight coefficient of $I$ adaptability criterion (the sum of all coefficients is 1 ); $r_{i}$-criteria of adaptability, based on [23], adapted for the subject area of EDMS and formalized in the notation of set theory: $r_{1}=\left(t_{\max }-t\left(S W^{*}, H W^{*}\right)\right) / t_{\max }$-time criterion from the user's system access to obtaining permission to work with it (authorization time), defined as the ratio of time in the current configuration $t\left(S W^{*}, H W^{*}\right)$ to the highest $t_{\max }$ of all options $S W, H W ; r_{2}=\left(n\left(M F^{*}\right) / n(M F)_{\max }\right)$ criterion of functionality, determined by the ratio of the number of implemented functional blocks $n\left(M F^{*}\right)$ to their maximum possible number $n(M F)_{\max }$; $r_{3}=\sum q g_{i} / n U, i=\overline{1, n U}$-flexibility criterion based on $q g i$ user assessment (quantity $n U$ ), and reflecting the ability of the interface to adapt to the needs of users with different equipment configurations; $r_{4}=(n T e s t-$ $n$ Error)/nTest-criterion of the interface security against errors made by the user, determined by the number of errors nError made during the tests nTest; $r_{5}=\sum q d_{i} / n U, i=\overline{1, n U}$-accessibility criterion, based on qgi user assessment (quantity $n U$ ), and reflecting the quality of navigation, ease of installation of EDMS, interface convenience from the point of text clarity, its size and design; $\quad r_{6}=\sum q k_{i} / n U, i=\overline{1, n U}$-comfort criterion of interaction with EDMS, based on $q k_{i}$ user assessment (quantity $n U$ ), reflecting the availability of the necessary auxiliary means of user support (search or reference subsystems), as well as user assessment on the speed of the system mastering. Criteria values range from 0 to 1 .

The developed model allows us to formalize the structure and correlation of EDMS components. The 
model novelty considers the criteria of EDMS adaptability with the calculation of economic costs, productivity and quality. The presented calculation formulas are aimed at obtaining an objective assessment of EDMS adaptivity.

\subsection{Two-Stage Formulation of the Problem of Adaptive EDMS Synthesis}

It is necessary to select optimization criteria [11] to state the problem of the adaptive EDMS design. Two approaches are possible: finding the optimal Pareto set for solving a multicriteria optimization problem or choosing a single criterion as the main one, transferring the others to a number of additional ones and using them as constraints.

The second method allows you to immediately find the only solution that is optimal by the main criterion and satisfies the admissible area for all other criteria. As the main criterion within the task, it is proposed to use the degree of adaptability of EDMS, since it has the decisive importance. However, it is necessary to take into account that the adaptability criterion is calculated for each user separately and it is impossible to obtain a universal solution suitable for all users. Therefore, the following approach is proposed: at the first stage it's necessary to carry out a structuralparametric synthesis of EDMS by the criterion of total discounted costs, and at the second stage we need to optimize the solution obtained by varying the interface parameters for each user by the adaptability criterion.

Thus, the formulation of the problem of designing an adaptive EDMS is formulated as follows.

At the first stage, it is necessary for EDMS with $U M V C$ architecture to determine such vector of variables $x^{*}=\left(M W^{*}, M F^{*}, H W^{*}, S W^{*}\right)$ from the set of permissible solutions $X$, at which the objective function of total discounted costs reaches its minimum:

$$
\left\{M W^{*}, M F^{*}, H W^{*}, S W^{*}\right\}=\underset{M W, M F, H W, S W}{\arg \min }\left(R_{V}\right)
$$

With the restrictions on:

- Minimum workflow performance:

$$
\begin{aligned}
R_{E} \geq \sum_{i=1}^{K} \frac{w_{i}}{t u_{i}}, 0 & <\sum_{j=1}^{J_{i}} t_{i j} \leq t u_{i}, \\
0 & <\sum_{j=1}^{J_{i}} t_{i j} \leq t u_{i},
\end{aligned}
$$

- Required quality of EDMS work:

$$
R_{Q} \geq R_{Q}^{*},
$$

- Minimal adaptability of EDMS based on expert assessment:

$$
R_{A} \geq R_{A}^{*},
$$

$t u_{i}$ - maximum document processing time; $R_{Q}{ }^{*}$ - basic value of assessing the quality of information system, determined by an expert group or an assessment of competitors' software; $R_{A}{ }^{*}$ - minimum value of adaptability assessment.

At the second stage, it is necessary to find such parameters of EDMS software $S W^{*}$, at which the objective adaptability function reaches its maximum.

$$
\left\{S W^{*}\right\}=\underset{S W}{\arg \max }\left(R_{A}\right),
$$

At fulfillment of the restrictions determined at the first stage of structural and parametric synthesis (20-23).

It should be noted that the second stage of the task can be automated through the use of machine learning methods. For this, it is necessary to implement a specialized neural network module of structuralparametric optimization $N N S P O$, which, based on the current structure $S M$ and parameters $P M$ of $E D M S$ modules, selects the optimal ones-SM ${ }^{*}$ and $P M^{*}$ accordingly. The optimality of the obtained values is determined by the above adaptive criterion $R_{A}$. Then we receive:

$$
\operatorname{NNSPO}\left(S M, P M, R_{A}\right)=\left(S M^{*}, P M^{*}\right)
$$

The advantages of this approach are its perspective and significant time savings due to the automation of the adaptation process-the problem of structuralparametric synthesis can be solved automatically and in a very short period of time with a trained neural network. The disadvantages of the approach at the current stage of development of computing technology are the lack of accuracy of neural networks in solving problems of similar complexity, the lack of a large amount of basic data necessary for high-quality training of neural networks. However, the constant growth of computational power, the development of machine learning technologies and the accumulation of data on the design process of EDMS will make it possible to successfully solve such problems in the near future [3].

\section{Discussions}

As a result of the conducted research, a two-stage task of structural-parametric synthesis of adaptive EDMS was set. Its distinctive feature is the division of the synthesis problem into two stages. The first stage defines the optimal solution according to the criterion of total discounted costs, which reflects the economic efficiency of EDMS. At the second stage, the optimization is carried out according to the criterion of adaptability of EDMS, which includes six components, that makes possible to obtain a comprehensive assessment of the system. The formulas are formalized and presented for each component of the criterion of adaptability. These formulas are also adapted to the subject area of EDMS. This provided the accurate calculation of EDMS adaptability degree.

It was necessary to develop original software based on the new UMVC of EDMS architecture to solve the 
problem. The use of paradigms and approaches from object-oriented programming in the studied area of electronic document management reduces the distance between the theoretical model and its practical implementation in the form of software. This question repeatedly arises when discussing mathematical models, since they are often very far from real software products and do not represent any benefit for software developers. That is why the development of UMVC of EDMS architecture took into account many of the rules and patterns in the programming field.

In addition to the classical formulation of the problem of structural-parametric synthesis in the framework of this study, the hypothesis about the possibility of applying the neural networks to solve the problem of structural-parametric synthesis of EDMS and its adaptation to changes in business processes was considered. The implementation of optimization modules based on neural networks is a promising direction from a scientific and practical point of view. Such modules can dynamically control the entire structure of EDMS in real time, can increase the flexibility of the EDMS architecture, make it dynamically changeable throughout the system's life cycle, and also allow it to be adapted to the individual characteristics of each user and increase its performance by optimizing the interface parameters for equipment characteristics of each client. Therefore, further work in this direction will include the development of the algorithmic support and software based on neural networks necessary for problem solving of EDMS adaption.

The scientific novelty of the proposed approach consists in the shift to the two-stage optimization task, the adaptive EDMS criteria formalization, the development of the architecture and mathematical model of adaptive EDMS based on MVC paradigm modernization.

\section{Conclusions}

This article discusses several important issues solved in the framework of the problem of structuralparametric synthesis of EDMS. First, the analysis of approaches for the consideration of adaptability factor of interface in the formalization of EDMS and its optimization solution was carried out. Secondly, it was determined that, based on the existing mathematical model of EDMS [11], it is impossible to effectively solve the problem of structural-parametric synthesis of an adaptive EDMS; a shift to a new type of structural and mathematical support is necessary. Therefore, this article discusses UMVC architecture, based on MVC design pattern, which is common in the field of programming [17]. However, the proposed architecture was expanded by including the User's entity, decomposition of Model into three key components, formalization of the functioning processes and evaluation of EDMS, and the study of the interface adaptation process, which suggests its originality.

Within the framework of the proposed architecture, the mathematical model of adaptive EDMS, its key components are considered in detail. Particular attention is paid to the evaluations of the information system for a number of indicators: total discounted costs, productivity, quality, adaptability. On the basis of the proposed model, a two-stage structuralparametric synthesis problem was set, which differs from the optimization problem considered in [11] by the criterion of total discounted costs, which reflects the economic efficiency of EDMS, and then by the adaptability criterion for each user. The possibility of using machine learning methods to simplify the process of EDMS adaption is considered.

The obtained scientific results can be applied in practical research on the optimization of EDMS, as well as used in solving problems of various information systems design.

This work was supported by the Ministry of Science and Higher Education of the Russian Federation within the grant of the President of the Russian Federation, MK-74.2020.9.

\section{References}

[1] Abrahamsson P., Salo O., Ronkainen J., and Warsta J., "Agile Software Development Methods: Review and Analysis," arXiv preprint arXiv:1709.08439, 2017.

[2] Bennaceur A. and Meinke K., Machine Learning for Dynamic Software Analysis: Potentials and Limits, Springer, 2018.

[3] Biamonte J., Wittek P., Pancotti N., Rebentrost P., Wiebe N. and Lloyd S., "Quantum machine learning. Nature," Nature Publishing Group, vol. 549, no. 7671, pp. 195-202, 2017.

[4] Da Cruz A., "Use Case and User Interface Patterns for Data Oriented Applications," in Proceedings of International Conference on Model-Driven Engineering and Software Development, Lisbon, pp. 117-133, 2014.

[5] Fernández-García A., Iribarne L., Corral A., Criado J., and Wang J., "A Recommender System for Component-Based Applications Using Machine Learning Techniques," Knowledge-Based Systems, vol. 164, pp. 68-84, 2019.

[6] Firmenich D., Firmenich S., Rossi G., Winckler M., and Distante D., "User Interface Adaptation Using Web Augmentation Techniques: Towards a Negotiated Approach," in Proceedings of International Conference on Web Engineering, Rotterdam, pp. 147-164, 2015.

[7] Karthiekheyan K., Ahmed I., and Jayalakshmi J., "Pair Programming for Software Engineering Education: An Empirical Study," The 
International Arab Journal of Information Technology, vol. 15, no. 2, pp. 246-255, 2018.

[8] Kashyap P., Machine Learning for Decision Makers, Apress, 2017.

[9] Katan S., Grierson M., and Fiebrink R., "Using Interactive Machine Learning to Support Interface Development through Workshops with Disabled People," in Proceedings of the $33^{\text {rd }}$ Annual ACM Conference on Human Factors in Computing Systems, New York, pp. 251-254, 2015.

[10] Krasnyanskiy M., Obukhov A., Solomatina1 E., Skvortsov V., and Khvorov V., "Formalization of Document Management Using Multilevel Graph Model of Information Processing," International Multidisciplinary Scientific Geo Conference, vol. 18, pp. 413-420, 2018.

[11] Krasnyanskiy M., Ostroukh A., Karpushkin S., and Obukhov A., "Formulation of the Problem of Structural and Parametric Synthesis of Electronic Document Management System of Research and Education Institution," Global Journal of Pure and Applied Mathematics, vol. 12, no. 3, pp. 2395-2409, 2016.

[12] Kultsova M., Potseluico A., Zhukova I., Skorikov A., and Romanenko R., "A Two-Phase Method of User Interface Adaptation for People with Special Needs," in Proceedings of Conference on Creativity in Intelligent Technologies and Data Science, Volgograd, pp. 805-821, 2017.

[13] Molnár B. and Benczúr A., "Modeling Information Systems from the Viewpoint of Active Documents," Vietnam Journal of Computer Science, vol. 2, no. 4, pp. 229-241, 2015.

[14] Ndadji M. and Tchendji M., "A Software Architecture for Centralized Management of Structured Documents in a Cooperative Editing Workflow," in Proceedings of Innovation and Interdisciplinary Solutions for Underserved Areas, Dakar, pp. 279-291, 2017.

[15] Paramonova I., "Electronic DocumentManagement Systems: A Classification and New Opportunities for A Scientific Technical Library," Scientific and Technical Information Processing, vol. 43, no. 3, pp. 136-143, 2016.

[16] Park K. and Lee S., Requirements Engineering in the Big Data Era, Springer, 2015.

[17] Reenskaug T., "Models-views-controllers" Technical Report, University of Oslo, 1979.

[18] Sen A., Ghosh S., Kundu D., Sarkar D., and Sil J., "Study of Engineered Features and Learning Features in Machine Learning-A Case Study in Document Classification," in Proceedings of International Conference on Intelligent Human Computer Interaction, Pilani, pp. 161-172, 2016.

[19] Shatnawi A. and Shatnawi R., "Generating A Language-Independent Graphical User Interfaces
From UML Models," The International Arab Journal of Information Technology, vol. 13, no. 3, pp. 291-296, 2016.

[20] Uqaili I. and Ahsan S., "Machine Learning Based Prediction of Complex Bugs in Source Code," The International Arab Journal of Information Technology, vol. 17, no. 1, pp. 26-37, 2020.

[21] Verlan A., Sopel M., and Furtat J., "About the Organization of the Adaptive User Interface in Automated Systems," Izvestiya SFedU. Engineering Sciences, vol. 1, no. 150, pp. 100$110,2014$.

[22] Wang H., Tudorache T., Dou D., Noy N., and Musen M., "Analysis and Prediction of User Editing Patterns in Ontology Development Projects," Journal on Data Semantics, vol. 4, no. 2, pp. 117-132, 2015.

[23] Yakovlev S. and Korsuntsev L., "Development of Adaptive Human-Machine Interface and the Criteria of Their Evaluation in Educational Systems," Educational Technologies and Society, vol. 1, no. 16, 547-563, 2013.

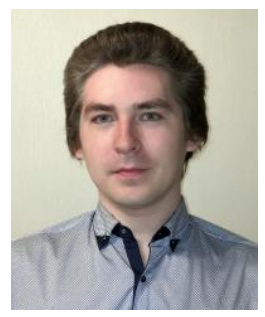

Artem Obukhov received his Ph.D. degree in technical sciences from Tambov State Technical University, Tambov, Russia, in 2016. Associate Professor. His research interests include computer-aided design of information systems, artificial intelligence, and system analysis.

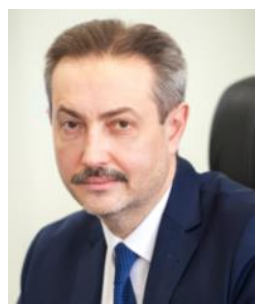

Mikhail Krasnyanskiy obtained Doctor of technical sciences in Processes and Apparatuses of Chemical Technologies, rector of Tambov State Technical University. His research interests include theory and methods of development of training and electronic document management systems.

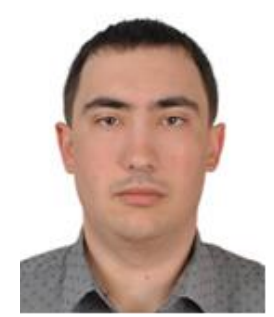

Denis Dedov received his Ph.D. degree in technical sciences from Tambov State Technical University, Tambov, Russia, in 2012. His research interests include developing of adapting training and information systems. 